TAIWANESE JOURNAL OF MATHEMATICS

Vol. 17, No. 1, pp. 239-257, February 2013

DOI: $10.11650 /$ tjm.17.2013.1913

This paper is available online at http://journal.taiwanmathsoc.org.tw

\title{
ON A GENERALIZATION OF LIFTING MODULES RELATIVE TO A TORSION THEORY
}

\author{
M. Tamer Koşan, Truong Cong Quynh and Yahya Talebi
}

\begin{abstract}
Let $\tau=(\mathcal{T}, \mathcal{F})$ be a torsion theory. An $R$-module $M$ is $\tau$-lifting, if for any submodule $N$ of $M$ there exists a decomposition $M=A \oplus B$ such that $A \leq N$ and $N \cap B$ is $\tau$-small in $M$. This definition unifies several definitions on generalizations of lifting property of modules. In the present paper, various results on $\tau$-lifting modules are developed, many extending known results.
\end{abstract}

\section{INTRODUCTION}

Throughout this paper, $R$ will always denote an associative ring with unity and all modules will be assumed to be unital right $R$-modules. The notions, " $\leq$ " will denote a submodule, " $\leq d$ " a module direct summand and " $\leq_{e}$ " an essential submodule.

Let $\tau=(\mathcal{T}, \mathcal{F})$ be a torsion theory. Then $\tau$ is uniquely determined by its associated class $\mathcal{T}$ of $\tau$-torsion modules $\mathcal{T}=\{M \in \operatorname{Mod}-R \mid \tau(M)=M\}$ where for a module $M, \tau(M)=\sum\{N \mid N \leq M, N \in \mathcal{T}\}$ and $\mathcal{F}$ is referred as $\tau$-torsion free class and $\mathcal{F}=\{M \in \operatorname{Mod}-R \mid \tau(M)=0\}$. A module in $\mathcal{T}$ (or $\mathcal{F}$ ) is called $\tau$-torsion module (or $\tau$-torsionfree module). Every torsion class $\mathcal{T}$ determines in every module $M$ a unique maximal $\mathcal{T}$-submodule $\tau(M)$, the $\tau$-torsion submodule of $M$, and $\tau(M / \tau(M))=0$. In what follows $\tau$ will represent a hereditary torsion theory, that is, if $\tau=(\mathcal{T}, \mathcal{F})$ then the class $\mathcal{T}$ is closed under taking submodules, direct sums, images and extensions by short exact sequences, equivalently the class $\mathcal{F}$ is closed under submodules, direct products, injective hulls and isomorphic copies. The torsion functor for the dual Goldie torsion theory will be denoted by $\tau_{*}$. Then the dual Goldie torsion theory $\tau_{*}=\left(\mathcal{T}_{*}, \mathcal{F}_{*}\right)$ is generated by the class of small $R$-modules.

For any right $R$-module $M$, a submodule $N$ of $M$ is said to be small in $M$, if $M \neq N+L$ for every proper submodule $L$ of $M$. Recently, two generalizations of small modules were introduced by Zhou [15].

Received April 13, 2012, accepted July 9, 2012.

Communicated by Bernd Ulrich.

2010 Mathematics Subject Classification: 16L30, 16E50.

Key words and phrases: $\tau$-Small submodule, $\tau$-Lifting module. 
A submodule $N$ of $M$ is said to be a $\delta$-small in $M$ if, whenever $N+X=M$ with $M / X$ singular, then $X=M$. Recall that an $R$-module module $M$ is called lifting if, for all $N \leq M$, there exists a decomposition $M=A \oplus B$ such that $A \leq N$ and $N \cap B$ is small in $M$. According to Koşan [5], a module $M$ is said to be $\delta$-lifting if, for all $N \leq M$, there exists a decomposition $M=A \oplus B$ such that $A \leq N$ and $N \cap B$ is $\delta$-small in $M$. Clearly, every lifting module is $\delta$-lifting and every singular $\delta$-lifting module is lifting. A submodule $L$ of $M$ is called a $\delta$-supplement of $N$ in $M$ if $N$ and $L$ satisfy one of the following conditions:

(i) $M=N+L$ and $N \cap L$ is $\delta$-small in $L$.

(ii) $M=N+L$ and for any proper submodule $K$ of $L$ with $L / K$ singular, $M \neq$ $N+K$.

A module $M$ is called a $\delta$-supplemented module if every submodule of $M$ has a $\delta$-supplement in $M$ (see [5]).

The torsion theory $\tau$ is assumed to be cohereditary, that is, we assume that homomorphic images of $\tau$-torsion free modules are $\tau$-torsion free. Let $N$ be a submodule of a module $M$. Then $N$ is called $\tau_{\mathcal{F}}$-small in $M$ if it is small in $M$ and $N \in \mathcal{F}$. In this case we write $N \ll_{\tau_{\mathcal{F}}} M$.

$\tau=(\mathcal{T}, \mathcal{F})$ be a hereditary torsion theory and $N$ be a submodule of an $R$-module $M$. $N$ is said to be a $\tau_{\mathcal{T}}$-small in $M$ if $M \neq N+Y$ for any proper submodule $Y$ of $M$ with $M / Y \in \mathcal{T}$. As Zhou pointed out in [15, Remark 5], in case $\tau$ is improper torsion theory or Goldie torsion theory, all results in [15] and [5] can be obtain similarly. Zhou raised the question whether these can be obtain for the setting of an arbitrary hereditary torsion theory or not. In this paper, we will give some answers to this question.

We will refer to [1], [3] and [12] for all undefined notions used in the text.

\section{2. $\tau$-LIFTING MODULES}

The properties of $\delta$-small modules that are listed in [15, Lemmas 1.2 and 1.3] also hold for $\tau$-small modules. We write them for convenience.

Lemma 2.1. Let $M$ be a module.

(1) For submodules $N, K, L$ of $M$ with $K \leq N$, we have

(a) $N \ll_{\tau_{\mathcal{T}}} M$ if and only if $K \ll_{\tau_{\mathcal{T}}} M$ and $N / K \ll_{\tau_{\mathcal{T}}} M / K$.

$\left(a^{\prime}\right) N \ll_{\tau_{\mathcal{F}}} M$ if and only if $K \ll_{\tau_{\mathcal{F}}} M$ and $N / K \ll_{\tau_{\mathcal{F}}} M / K$.

(b) $N+L \ll_{\tau_{\mathcal{T}}} M$ if and only if $N \ll_{\tau_{\mathcal{T}}} M$ and $L \ll_{\tau_{\mathcal{T}}} M$.

$\left(b^{\prime}\right) N+L \ll_{\tau_{\mathcal{F}}} M$ if and only if $N \ll_{\tau_{\mathcal{F}}} M$ and $L \ll_{\tau_{\mathcal{F}}} M$.

(2) If $K \ll_{\tau_{\mathcal{T}}} M$ and $f: M \rightarrow N$ is a homomorphism, then $f(K) \ll_{\tau_{\mathcal{T}}} N$. In particular, if $K \ll_{\tau_{\mathcal{T}}} M \leq N$, then $K \ll_{\tau_{\mathcal{T}}} N$ 
(3) Let $K_{1} \leq M_{1} \leq M, K_{2} \leq M_{2} \leq M$ and $M=M_{1} \oplus M_{2}$. Then $K_{1} \oplus K_{2} \ll_{\tau_{\mathcal{T}}}$ $M_{1} \oplus M_{2}$ if and only if $K_{1} \ll_{\tau_{\mathcal{T}}} M_{1}$ and $K_{2} \ll_{\tau_{\mathcal{T}}} M_{2}$. In particular, if $K \leq L \leq_{d} M$ and $K \ll_{\tau_{\mathcal{T}}} M$ then $K \ll_{\tau_{\mathcal{T}}} L$.

Example 2.2. Let $\tau=(\mathcal{T}, \mathcal{F})$ be a torsion theory, $M$ an $R$-module and $N$ be a submodule of $M$.

(1) $N \ll_{\tau_{\mathcal{T}}} M$ if and only if $N \ll M$, whenever $M=N+X$ with $M / X \in \mathcal{T}$, we have $M=X$.

(2) Assume that $\tau(N)=N$. Then $N \ll_{\tau_{\mathcal{T}}} M$ if and only if $N \ll M$.

Let $\tau=(\mathcal{T}, \mathcal{F})$ be a torsion theory and $M$ an $R$-module. Let $B \leq A \leq M$, if $A / B \ll_{\tau_{\mathcal{T}}} M / B\left(A / B \ll_{\tau_{\mathcal{F}}} M / B\right)$, then $B$ is called a $\tau_{\mathcal{T}^{-} \text {-cosmall }}\left(\tau_{\mathcal{F}^{-}}\right.$-cosmall $)$ submodule of $A$ in $M$. A submodule $A$ of $M$ is called $\tau_{\mathcal{T}}$-coclosed $\left(\tau_{\mathcal{F}}\right.$-coclosed $)$ if $A$ has no proper $\tau_{\mathcal{T}}$-cosmall $\left(\tau_{\mathcal{F}}\right.$-cosmall $)$ submodule.

\section{Remark 2.3.}

(1) If $N$ is coclosed in $M$, then $N$ is a $\tau_{\mathcal{T}}$-coclosed submodule of $M$.

$\left(1^{\prime}\right)$ If $N$ is coclosed in $M$, then $N$ is a $\tau_{\mathcal{F}}$-coclosed submodule of $M$.

(2) Every $\tau$-torsion, $\tau_{\mathcal{T}}$-coclosed submodule $N$ of a module $M$ is coclosed in $M$.

$\left(2^{\prime}\right)$ Every $\tau$-torsion free, $\tau_{\mathcal{F}}$-coclosed submodule $N$ of a module $M$ is coclosed in $M$.

Proof. (2') Suppose that $K \leq N \leq M$ and $N / K \ll M / K$. We must show that $N=K$. Since $N \in \mathcal{F}$, we get $N / K \in \mathcal{F}$. Therefore $N / K \ll_{\tau_{\mathcal{F}}} M / K$, but $N$ is $\tau_{\mathcal{F}}$-coclosed, thus $N=K$.

We give some important fundamental properties of $\tau_{\mathcal{T}}$ and $\tau_{\mathcal{F}}$-coclosed submodules.

Lemma 2.4. Let $K \leq L \leq M$. Then the following hold.

(1) If $L$ is $\tau_{\mathcal{T}}$-coclosed in $M$, then $L / K$ is $\tau_{\mathcal{T}}$-coclosed in $M / K$.

$\left(1^{\prime}\right)$ If $L$ is $\tau_{\mathcal{F}}$-coclosed in $M$, then $L / K$ is $\tau_{\mathcal{F}}$-coclosed in $M / K$.

(2) If $L \leq M$ is $\tau_{\mathcal{T}}$-coclosed, then $K \ll_{\tau_{\mathcal{T}}} M$ implies $K \ll_{\tau_{\mathcal{T}}} L$.

(3) If $K$ is $\tau_{\mathcal{T}}$-coclosed in $M$, then $K$ is $\tau_{\mathcal{T}}$-coclosed in $L$ and the converse is true if $L$ is $\tau_{\mathcal{T}}$-coclosed in $M$.

$\left(3^{\prime}\right)$ If $K$ is $\tau_{\mathcal{F}}$-coclosed in $M$, then $K$ is $\tau_{\mathcal{F}}$-coclosed in $L$ and the converse is true if $L$ is $\tau_{\mathcal{F}}$-coclosed in $M$.

\section{Proof.}

(1) Suppose there exists a proper submodule $N$ of $L$ such that $N / K \leq L / K$ is $\tau_{\mathcal{T}}$-cosmall in $M / K$. Then $(L / K) /(N / K) \ll_{\tau_{\mathcal{T}}}(M / K) /(N / K)$, and so $L / N \ll_{\tau_{\mathcal{T}}} M / N$. This contradicts the assumption that $L$ is $\tau_{\mathcal{T}}$-coclosed in $M$. 
$\left(1^{\prime}\right)$ It is similarly.

(2) Consider $K \leq L$ with $K \ll_{\tau} M$. Assume $K+K^{\prime}=L$ for some $K^{\prime} \subseteq L$, with $L / K^{\prime} \in \mathcal{T}$. Choose $K^{\prime} \subset L^{\prime} \subset M$ such that $M / K^{\prime}=L / K^{\prime}+L^{\prime} / K^{\prime}$ and $\left(M / K^{\prime}\right) /\left(L^{\prime} / K^{\prime}\right) \in \mathcal{T}$. Then $M=L+L^{\prime}=K+K^{\prime}+L^{\prime}=L^{\prime}$, and this shows that $L / K^{\prime} \ll_{\tau_{\mathcal{T}}} M / K^{\prime}$. Since $L$ is $\tau_{\mathcal{T}^{-}}$-coclosed in $M$, we conclude $L=K^{\prime}$ and so $K \ll_{\tau_{\mathcal{T}}} L$.

(3) Assume that there exists $X \leq K$ such that $K / X \ll_{\tau_{\mathcal{T}}} L / X$. Then $K / X \ll_{\tau_{\mathcal{T}}}$ $M / X$. But $K$ is $\tau_{\mathcal{T}}$-coclosed in $M$ implies $K=X$. Hence $K$ is $\tau_{\mathcal{T}}$-coclosed in $L$.

Now suppose $K$ is $\tau_{\mathcal{T}}$-coclosed in $L$ and $L$ is $\tau_{\mathcal{T}}$-coclosed in $M$. Let $X \leq$ $K$ with $K / X \ll_{\tau} M / X$. By (1), $L / X$ is $\tau_{\mathcal{T}}$-coclosed in $M / X$ and by (2), $K / X \ll_{\tau_{\mathcal{T}}} L / X$. As $K$ is $\tau_{\mathcal{T}}$-coclosed in $L$, we can obtain that $X=K$.

$\left(3^{\prime}\right)$ It is similarly.

A submodule $K$ of $M$ is said to be a $\tau_{\mathcal{T}}$-supplement $\left(\tau_{\mathcal{F}}\right.$-supplement) provided there exists some $N \leq M$ such that $N+K=M$ and $N \cap K \ll_{\tau_{\mathcal{T}}} K\left(N \cap K \ll_{\tau_{\mathcal{F}}} K\right)$. A module $M$ is said to be a $\tau_{\mathcal{T}}$-supplemented ( $\tau_{\mathcal{F}}$-supplemented) module if every submodule of $M$ has a $\tau_{\mathcal{T}}$-supplement ( $\tau_{\mathcal{F}}$-supplement) in $M$.

Proposition 2.5. Let $M$ be a module and $N \leq M$. Consider the following conditions:

(1) $N$ is a $\tau_{\mathcal{T}}$-supplement submodule of $M$;

(2) $N$ is $\tau_{\mathcal{T}}$-coclosed in $M$;

(3) For all $X \leq N, X \ll_{\tau_{\mathcal{T}}} M$ implies $X \ll_{\tau_{\mathcal{T}}} N$.

Then $(2) \Rightarrow(3)$ holds. If $N$ is $\tau$-torsion, then (1) $\Rightarrow(2)$.

Proof. $\quad(2) \Rightarrow(3)$ Let $N$ be $\tau_{\mathcal{T}}$-coclosed in $M, X \leq N$ and $X \ll_{\tau_{\mathcal{T}}} M$. Assume that $Y \leq N$ and $N=X+Y$ such that $N / Y \in \mathcal{T}$. Now we want to show that $N / Y \ll_{\tau_{\mathcal{T}}} M / Y$. Let $M / Y=N / Y+H / Y$ with $Y \leq H \leq M$ such that $M / H \simeq(M / Y) /(H / Y) \in \mathcal{T}$. Then $M=N+H=X+Y+H=X+H$ implies $M=H$. Therefore $N / Y \ll_{\tau_{\mathcal{T}}} M / Y$. Since $N$ is $\tau_{\mathcal{T}}$-coclosed in $M$, we can obtain that $N=Y$. Hence $X \ll_{\tau_{\mathcal{T}}} N$.

(1) $\Rightarrow(2)$ Let $N$ be a $\tau$-torsion and $\tau_{\mathcal{T}}$-supplement of $K$ in $M$. Then $M=N+K$ and $N \cap K \ll_{\tau_{\mathcal{T}}} N$. Let $N^{\prime} \leq N$ and $N / N^{\prime} \ll_{\tau_{\mathcal{T}}} M / N^{\prime}$. Then $M / N^{\prime}=(K+$ $\left.N^{\prime}\right) / N^{\prime}+N / N^{\prime}$. Since $\left(M / N^{\prime}\right) /\left(K+N^{\prime}\right) / N^{\prime} \simeq M /\left(K+N^{\prime}\right)=(K+N) /\left(K+N^{\prime}\right)$ and $(K+N) /\left(K+N^{\prime}\right)$ is a homomorphic image of $N / N^{\prime}$, we can obtain that $(K+$ $N) /\left(K+N^{\prime}\right)$ is $\tau$-torsion. Therefore $M / N^{\prime}=\left(K+N^{\prime}\right) / N^{\prime}$, and so $M=K+N^{\prime}$. Then $N=(N \cap K)+N^{\prime}$. Since $N / N^{\prime} \in \mathcal{T}$ and $N \cap K \ll_{\tau_{\mathcal{T}}} N$, we have $K=K^{\prime}$. Hence $N$ is $\tau_{\mathcal{T}}$-coclosed in $M$. 
It is easy to see that implication $(2) \Rightarrow(3)$ holds for also $\tau_{\mathcal{F}}$-supplements and $\tau_{\mathcal{F}}$-coclosed submodules.

Lemma 2.6. If $K$ and $N$ are submodules of a module $M$, then $K$ is a $\tau_{\mathcal{F}^{-}}$ supplement of $N$ in $M$ if and only if $K$ is supplement of $N$ in $M$ and $K \cap N \in \mathcal{F}$.

Proof. The sufficiently is clear from the definitions. For the necessity, let

$$
\mathcal{A}=\{A \leq M \mid A+N=M\} \text { and } \mathcal{B}=\{B \leq M \mid B+N=M, B \cap N \in \mathcal{F}\} .
$$

Suppose that $K$ is minimal in $\mathcal{B}$. We want to show that $K$ is minimal in $\mathcal{A}$. Clearly $\mathcal{A} \subseteq \mathcal{B}$ and so $K \in \mathcal{A}$. Now if $A \in \mathcal{A}$ with $A \leq K$, then $K \cap N \in \mathcal{F}, A \cap N \leq$ $K \cap N \leq M$. Note that $A \cap N \in \mathcal{F}$. Therefore $A \in \mathcal{B}$ and by minimality of $K$ in $\mathcal{B}$, we have $K=A$. Thus $K$ is minimal in $\mathcal{A}$ as required.

Proposition 2.7. The following statements hold for an R-module $M$.

(1) If $K$ is a supplement of $N$ in $M$ such that either $K \in \mathcal{F}$ or $N \in \mathcal{F}$, then $K$ is a $\tau_{\mathcal{F}}$-supplement of $N$ in $M$.

(2) If $N \ll M$ and $N \notin \mathcal{F}$, then $N$ has no $\tau_{\mathcal{F}}$-supplement in $M$.

(3) Let $\tau=(\mathcal{T}, \mathcal{F})$ be a hereditary torsion theory and $M \in \mathcal{T}$. Then every nonzero small submodule $N$ of $M$ has no $\tau_{\mathcal{F}}$-supplement in $M$.

(4) If one of the following two conditions holds, then $N \cap K \ll_{\tau_{\mathcal{F}}} M$ and $K$ is $\tau_{\mathcal{F}}$-coclosed in $M$ :

(i) $K$ is a supplement of $N$ in $M$, and either $N$ or $K$ is $\tau$-torsion free.

(ii) $K$ is a $\tau_{\mathcal{F}}$-supplement of $N$ in $M$.

Proof.

(1) If either $K \in \mathcal{F}$ or $N \in \mathcal{F}$, then $K \cap N \in \mathcal{F}$. Therefore, by Lemma 2.6, $K$ is a $\tau_{\mathcal{F}}$-supplement of $N$ in $M$.

(2) Suppose to the contrary that $K$ is a $\tau_{\mathcal{F}}$-supplement of $N$ in $M$. Then $K$ is a supplement of $N$ and, since $N \ll M$, we get $K=M$. But $N=N \cap K \in \mathcal{F}$, giving the required contradiction.

(3) By (2), it suffices to show that $N \notin \mathcal{F}$. Suppose instead that $N \in \mathcal{F}$. Since $\tau$ is hereditary and $M \in \mathcal{T}$, we have $N \in \mathcal{T}$. Hence $N \in \mathcal{T} \cap \mathcal{F}=0$ and so $N=0$, a contradiction.

(4) Since (i) implies (ii) by (1), it suffices to assume (ii). By [14, 14.1], $N \cap K \ll M$. Furthermore, $N \cap K \in \mathcal{F}$ because $K$ is a $\tau_{\mathcal{F}}$-supplement of $N$. Consequently $N \cap K \ll_{\tau_{\mathcal{F}}} M$.

Let $K$ be a supplement of $L$ in $M$. Then by [4, Lemma 1.1], $K$ is coclosed in $M$ and so $\tau_{\mathcal{F}}$-coclosed in $M$ by Remark (1).

Now we give the proof of Proposition $2.5(1) \Rightarrow(2)$ for $\tau_{\mathcal{F}^{-}}$-supplement and $\tau_{\mathcal{F}^{-}}$ coclosed submodules. 
Proposition 2.8. Let $M$ be an R-module.

(1) If $N$ is a $\tau_{\mathcal{F}}$-supplement submodule of $M$, then $N$ is $\tau_{\mathcal{F}}$-coclosed in $M$.

(2) Let $K$ be a submodule of $M$ and $L$ be a $\tau_{\mathcal{F}}$-supplement of $K$ in $M$. Then $K$ is $\tau_{\mathcal{F}}$-coclosed in $M$ if and only if $K$ is a $\tau_{\mathcal{F}}$-supplement of $L$ in $M$.

(3) If $K$ has a $\tau_{\mathcal{F}}$-supplement in $M$ and $K$ is $\tau_{\mathcal{F}}$-coclosed in $M$, then $K$ is coclosed in $M$.

Proof.

(1) It follows from Proposition 2.7(4).

(2) Suppose that $K$ is $\tau_{\mathcal{F}}$-coclosed in $M$. Let $\mathcal{C}=\{C \leq M \mid C+L=M$ and $C \cap L \in \mathcal{F}$ \}. Clearly $K \in \mathcal{C}$, since $L$ is a $\tau_{\mathcal{F}}$-supplement of $K$. We must show that $K$ is minimal in $\mathcal{C}$. Let $N \in \mathcal{C}$ with $N \leq K$. Since $K$ is $\tau_{\mathcal{F}}$-coclosed in $M$, it suffices to show that $K / N \ll_{\tau_{\mathcal{F}}} M / N$. Since $N \in \mathcal{C}$, we have $M=N+L$, so $K=N+(L \cap K)$. As $K \cap L \in \mathcal{F}, K / N=(N+(K \cap L)) / N \in \mathcal{F}$. Now we show that $K / N \ll M / N$. Let $N \leq T \leq M$ and $K / N+T / N=M / N$. Then $K+T=M$. On the other hand $M=N+L$ implies that $T=N+(L \cap T)$, therefore $M=K+(L \cap T)$. Since $L$ is a $\tau_{\mathcal{F}}$-supplement and thus a supplement of $K$, we have $L \cap T=L$, then $L \leq T$. As $T+L=M, T=M$. Therefore $T / N=M / N$.

Assume that $K$ is a $\tau$-supplement of $L$ in $M$. By Lemma 2.6, $K$ is a supplement of $L$ in $M$. Then by [4, Lemma 1.1], $K$ is coclosed. By Remark 2.3, $K$ is $\tau_{\mathcal{F}^{-}}$ coclosed in $M$.

(3) It follows from Lemma 2.6 and (2).

The following lemma can be seen by the proof of the [15, Lemma 1.2].

Lemma 2.9. Let $\tau_{*}=\left(\mathcal{T}_{*}, \mathcal{F}_{*}\right)$ be a Goldie torsion theory, $M$ an $R$-module and $N$ be a submodule of $M$. Then $N \ll_{\tau_{*}} M$ if and only if, whenever $M=X+N$ then $M=X \oplus Y$ for a projective semisimple submodule $Y$ with $Y \subseteq N$. In particular, there exists $P$ such that $M /(N \oplus P) \in \mathcal{T}_{*}$.

Let $\tau=(\mathcal{T}, \mathcal{F})$ be a torsion theory, $M$ an $R$-module. We call $M$ a $\tau_{\mathcal{T}^{-}}$-lifting $\left(\tau_{\mathcal{F}^{-}}\right.$ lifting) module if for any submodule $N$ of $M$ there exists a decomposition $M=A \oplus B$ such that $A \leq N$ and $N \cap B$ is $\tau_{\mathcal{T}}$-small ( $\tau_{\mathcal{F}}$-small) in $M$.

Example 2.10.

(1) Every lifting module is $\tau_{\mathcal{T}}$-lifting. Also every $\tau_{\mathcal{F}}$-lifting module is lifting.

(2) If $M$ is almost $\tau$-torsion free lifting module, then $M$ is $\tau_{\mathcal{F}}$-lifting.

(3) Let $\tau_{*}=\left(\mathcal{T}_{*}, \mathcal{F}_{*}\right)$ be a Goldie torsion theory and $M$ be an $R$-module.

(i) Every $\tau_{\mathcal{T}_{*}}$-lifting module which does not have any non-zero projective simple submodule is lifting. 
(ii) If $M$ has a unique decomposition series $M \supset U \supset V \supset(0)$, then $M \oplus$ $(U / V)$ is not $\tau_{\mathcal{T}_{*}}$-lifting.

Proof. (1) and (2) follow from definitions.

(3) (i) Let $M$ be a $\tau_{\mathcal{T}_{*}}$-lifting module and let $N \leq M$. Then there exists $A \leq N$ such that $M=A \oplus B$ and $N \cap B$ is $\tau_{\mathcal{T}_{*}}$-small in $B$. Assume that $B=(N \cap B)+L$ for some $L \leq N$. By Lemma 2.9, $B=Y \oplus L$ for some projective semisimple submodule $Y$ with $Y \leq N \cap B$. By hypothesis, $Y=0$. It implies that $B=L$ and so $N \cap B \ll B$. Hence $M$ is lifting.

(ii) Let $N=\{(u, \bar{u}): u \in U\} \leq M \oplus(U / V)$. Then $M \oplus(U / V)=(M \oplus(0))+N$ and $(M \oplus(U / V)) /(M \oplus(0)) \in \mathcal{T}_{*}$. Hence $N$ is not $\tau$-small in $M$. It is easily seen that $N$ is not a direct summand of $M \oplus(U / V)$ and $A=\{(v, \overline{0}): v \in V\}$ is the only proper submodule of $N$ which is not a direct summand of $M \oplus(U / V)$. Hence $M \oplus(U / V)$ is not $\tau_{\mathcal{T}_{*}}$-lifting.

Proposition 2.11. Let $\tau=(\mathcal{T}, \mathcal{F})$ be a torsion theory.

(1) The following are equivalent for a module $M$ :

(a) $M$ is $\tau_{\mathcal{T}}$-lifting (or $\tau_{\mathcal{F}}$-lifting).

(b) For all $N \leq M$, there exists a decomposition $N=A \oplus B$ such that $A$ is a direct summand of $M$ and $B \ll_{\tau_{\mathcal{T}}} M$ (or $B \ll_{\tau_{\mathcal{F}}} M$ ).

(c) For all $N \leq M$, there exists a direct summand $A$ of $M$ such that $A \leq N$ and $N / A \ll_{\tau_{\mathcal{T}}} M / A$ (or $\left.N / A \ll_{\tau_{\mathcal{F}}} M / A\right)$.

(d) For every submodule $N$ of $M$, there exists an idempotent homomorphism e from $M$ to $N$ such that $(1-e) N$ is $\tau_{\mathcal{T}}$-small (or $\tau_{\mathcal{F}}$-small).

(2) Assume that $M$ is an almost $\tau$-torsion module. Then the class of $\tau_{\mathcal{T}}$-lifting modules is closed under taking direct summands.

(3) Assume that $M$ is an almost $\tau$-torsion free module. Then the class of $\tau_{\mathcal{F}}$-lifting modules is closed under taking direct summands.

Proof. (1) This is standard.

(2) and (3) follow from definitions and Remark 2.3.

Example 2.12. Let $R$ denote the ring of all upper triangular $2 \times 2$ matrices with entries in the field $F$. Let $M$ denote the right $R$-module $M=\left(\begin{array}{cc}0 & F \\ F & F\end{array}\right)$. By [9, Theorem 4.41], $M$ is a lifting module.

(1) Let $X=\left(\begin{array}{cc}F & F \\ 0 & 0\end{array}\right)$. Then $X$ is an idempotent ideal of $R$. Clearly, $\tau_{X}$ is a hereditary torsion theory with torsion class $\tau=\{N \in \operatorname{Mod}-R \mid N X=0\}$. Note that, all proper submodules of $M$ are $N_{1}=\left(\begin{array}{cc}0 & F \\ 0 & F\end{array}\right)=\tau_{X}(M), N_{2}=\left(\begin{array}{cc}0 & F \\ 0 & 0\end{array}\right)=$ 
$\tau_{X}\left(N_{2}\right), N_{3}=\left(\begin{array}{cc}0 & 0 \\ 0 & F\end{array}\right)=\tau_{X}\left(N_{3}\right)$ and $N_{4}=\left(\begin{array}{cc}0 & 0 \\ F & F\end{array}\right)$. Since $N_{2}$ is a direct summand of $M, N_{1}=N_{2} \oplus N_{3}$ and $N_{3}$ is $\tau$-small in $M$, then $M$ is $\tau$-lifting module by Proposition 2.10 and Lemma 2.5(1).

(2) Let $e_{i j}$ the matrix units in $R$ and $Y=e_{12} R+e_{22} R$. Then $Y$ is an idempotent ideal of $R$ and so defines a hereditary torsion theory $\tau_{Y}$ with torsion class $\tau=\{K \in$ $M o d-R \mid K Y=0\}$. Let $A=e_{12} R$. Then $A$ is not direct summand of $M$. Since $A Y=e_{12} R$, then $A$ is not $\tau_{Y}$-torsion. Note that $A$ is a simple module. So $A$ does not contain any submodule $B$ such that $B$ is a direct summand of $M$ and $A / B$ is $\tau_{Y}$-torsion. Thus $M$ is not $\tau_{Y}$-lifting.

Recall that nonzero module $M$ is called hollow (or we say $\tau_{\mathcal{F}}$-hollow) if every proper submodule of $M$ is small (or $\tau_{\mathcal{F}^{-}}$-small in $M$ ). It is easy to see that $M$ is $\tau_{\mathcal{F}}$-hollow if and only if $M$ is a hollow almost $\tau$-torsion free module.

Proposition 2.13. The following are equivalent for a module $M$.

(1) $M$ is $\tau_{\mathcal{F}}$-hollow.

(2) $M$ is indecomposable $\tau_{\mathcal{F}}$-lifting.

(3) $M$ is lifting, indecomposable and almost $\tau$-torsion free.

(4) $M$ is $\tau_{\mathcal{F}}$-lifting, indecomposable and almost $\tau$-torsion free.

Proof. $\quad(1) \Rightarrow(2)$ If $M$ is $\tau_{\mathcal{F}}$-hollow, then it is hollow. This implies that $M$ is lifting (hence $\tau_{\mathcal{F}}$-lifting) and indecomposable.

$(2) \Rightarrow(3)$. If $M$ is $\tau_{\mathcal{F}}$-lifting then $M$ is lifting. Let $N \lesseqgtr M$. $M$ has a decomposition $M=N_{1} \oplus N_{2}$ such that $N / N_{1} \ll_{\tau_{\mathcal{F}}} M / N_{1}$. As $M$ is indecomposable, then $N_{1}=0$. Thus $N \ll_{\tau_{\mathcal{F}}} M$ follows that $N \in \mathcal{F}$. Therefore $M$ is almost $\tau$-torsion free.

$(3) \Rightarrow(4)$ This is trivial.

(4) $\Rightarrow(1)$ If $N \lesseqgtr M$ then, since $M$ is almost $\tau$-torsion free, $N \in \mathcal{F}$. Moreover, we have $N / N^{*} \ll_{\tau_{\mathcal{F}}} M / N^{*}$ for some direct summand $N^{*}$ of $M$. Since $N^{*} \lesseqgtr M$ and $M$ is indecomposable, we have $N^{*}=0$. Therefore $N \ll_{\tau_{\mathcal{F}}} M$ and thus $M$ is $\tau_{\mathcal{F}}$-hollow.

By a class $\mathcal{X}$ of $R$-modules we mean a collection of $R$-modules containing the zero module and closed under isomorphisms, i.e., any module isomorphic to some module in $\mathcal{X}$ also belongs to $\mathcal{X}$. By an $\mathcal{X}$-module we mean any member of $\mathcal{X}$, and a submodule $N$ of a module $M$ is called $\mathcal{X}$-submodule of $M$ if $N$ is a $\mathcal{X}$-module. Koşan and Harmanci [7] introduced $\mathcal{X}$-lifting module. The module $M$ is said to be a $\mathcal{X}$-lifting module if for every $\mathcal{X}$-submodule $N$ of $M$ there exists $A \leq N$ such that $M=A \oplus B$ and $N \cap B \ll B$ (see also [8] and [2]).

\section{Example 2.14.}

(1) Let $\zeta=(0, \operatorname{Mod}-R)$ where 0 denotes the class of zero modules. Clearly, every an $R$-module $M$ is $\tau$-lifting relative to a torsion theory $\zeta$, i.e., $M$ is $\zeta$-lifting if and only if $M$ is semisimple. 
(2) An $R$-module $M$ is $\chi$-lifting if and only if it is lifting, where $\chi$ is the torsion theory in which every module is considered to be torsion.

Lemma 2.15. We consider the following commutative diagram of right R-modules;

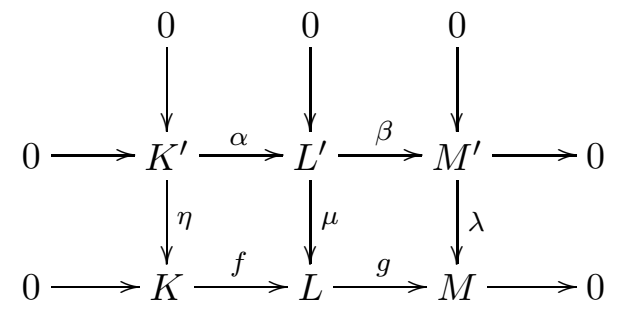

where $\eta\left(K^{\prime}\right) \ll_{\tau_{\mathcal{T}}} K$ and $\lambda\left(M^{\prime}\right) \ll_{\tau_{\mathcal{T}}} M$. If $M$ is a projective module then $\mu\left(L^{\prime}\right) \ll_{\tau_{\mathcal{T}}} L$.

Proof. Assume that $K^{\prime} \leq L^{\prime}, K^{\prime} \leq K, L^{\prime} \leq L, M^{\prime} \leq M$ and $K \leq L$ without loss of generality. Hence $\eta, \mu, \lambda$ and $\alpha, \beta$ are inclusion homomorphism. Clearly, $K^{\prime} \ll_{\tau_{\mathcal{T}}} L$ and $L^{\prime} / K^{\prime} \cong M^{\prime} \ll_{\tau_{\mathcal{T}}} M$. Since $K^{\prime} \subseteq \operatorname{Ker}(g)$ and $M$ is a projective module, it is easy to see that $M$ is a direct summand of $L / K^{\prime}$. Then we have $L^{\prime} / K^{\prime} \ll_{\tau_{\mathcal{T}}} L / K^{\prime}$ and so $L^{\prime} \ll_{\tau} L$.

Recall that a projective module $P$ is called a projective $\tau$-cover of a module $M$ if there exists an epimorphism $f: P \longrightarrow M$ with $\operatorname{Ker}(f) \ll_{\tau_{\mathcal{T}}} M$. A right $R$-module is said to be a $\tau$-perfect if $M$ possesses a projective $\tau$-cover. So a ring $R$ is called $\tau$-perfect if every right $R$-module is $\tau$-perfect (see [15]).

Proposition 2.16. Let $\mathcal{P}$ be any class of $\tau$-perfect $R$-modules. Then $\mathcal{P}$ is closed under extensions.

Proof. Let $0 \rightarrow K \stackrel{f}{\rightarrow} L \stackrel{g}{\rightarrow} \quad M \rightarrow 0$ be a short exact sequence such that $K, M$ are $\tau$-perfect modules. We have the following commutative diagram; where all rows and columns are exact, $P_{1}, P_{2}, P_{3}$ are projective modules with $P_{1}, P_{3} \tau$-covers of $K, M$, respectively. By Lemma 2.15, $P_{2}$ is a projective $\tau$-cover of $L$.

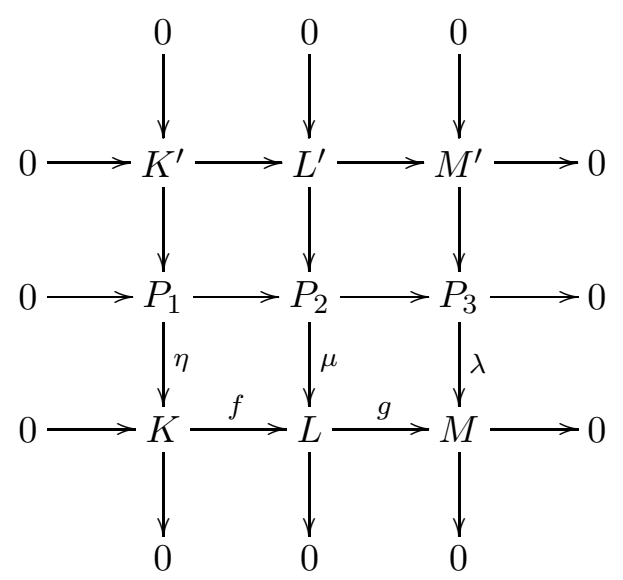


Let $\mathcal{M}$ denote the class of all $R$-modules. Then a module $M$ is lifting if and only if $M$ is $\mathcal{M}$-lifting.

Theorem 2.18. Let $\mathcal{P}$ be any class of $\tau$-perfect $R$-modules.

(1) $R$ is semisimple if and only if $\mathcal{P}=\{M: M$ is semisimple right $R$-module $\}$.

(2) If $R$ is semisimple then $M$ is lifting if and only if $M$ is $\mathcal{P}$-lifting.

Proof. Clear.

\section{Decompositions of $\tau$-Lifting Modules}

In Proposition 2.11, we proved that the class of $\tau_{\mathcal{T}}$-lifting modules is closed under direct summands. But it is not closed under factor modules or direct sums. First, we give sufficient conditions for a factor module of a $\tau_{\mathcal{T}}$-lifting module to be $\tau_{\mathcal{T}}$-lifting and for a direct sum of two $\tau_{\mathcal{T}}$-lifting modules to be $\tau_{\mathcal{T}}$-lifting.

A submodule $X$ of a module $M$ is called fully invariant if for every $h \in \operatorname{End}_{R}(M)$, $h(X) \subseteq X . M$ is said to be a duo module if every submodule of $M$ is fully invariant.

Proposition 3.1. Let $\tau=(\mathcal{T}, \mathcal{F})$ be a torsion theory and $M=M_{1} \oplus M_{2} a$ duo module. If $M_{1}$ and $M_{2}$ are almost $\tau$-torsion free modules, then $M$ is an almost $\tau$-torsion free module.

Proof. Let $N$ be a submodule of $M$. Then $N=\left(M_{1} \cap N\right) \oplus\left(M_{2} \cap N\right)$. Since $M_{i} \cap N \lesseqgtr M_{i}$ and each $M_{i}$ is almost $\tau$-torsion free module, for $i=1,2$, we can obtain that $M_{i} \cap N \in \mathcal{F}$. As $\mathcal{F}$ is closed under direct sum, $N \in \mathcal{F}$.

A module $M$ is called distributive if its lattice of submodules is a distributive lattice, that is $A \cap(B+C)=(A \cap B)+(A \cap C)$ for any submodules $A, B$ and $C$ of M.

Proposition 3.2. Let $\tau=(\mathcal{T}, \mathcal{F})$ be a torsion theory, $M$ a $\tau_{\mathcal{T}}$-lifting module and $X \leq M$. Then $M / X$ is $\tau_{\mathcal{T}}$-lifting in each of the following cases:

(1) For every direct summand $K$ of $M,(K+X) / X$ is a direct summand of $M / X$.

(2) $M$ is a distributive module.

(3) For all $e^{2}=e \in \operatorname{End}(M)$, eX $\subseteq X$. In particular, $X$ is a fully invariant submodule of $M$.

Proof.

(1) Let $A / X \leq M / X$. Since $M$ is $\tau_{\mathcal{T}}$-lifting, there exists a direct summand $K$ of $M$ such that $K \subseteq A$ and $A / K$ is $\tau_{\mathcal{T}}$-small in $M / K$ by Proposition 2.5. By hypothesis, $(K+X) / X$ is a direct summand of $M / X$. Clearly, $(K+X) / X \subseteq$ $A / X$. Now $A /(K+X)$ is $\tau_{\mathcal{T}}$-small in $M /(K+X)$ by Lemma 2.1. Hence $M / X$ is $\tau_{\mathcal{T}}$-lifting. 
(2) Let $M=K \oplus L$. Then $M / X=[(K+X) / X]+[(L+X) / X]$ and $X=$ $X+(K \cap L)=(X+K) \cap(X+L)$. So, $M / X=[(K+X) / X] \oplus[(L+X) / X]$. By $(1), M / X$ is $\tau_{\mathcal{T}}$-lifting.

(3) Let $M=K \oplus L$. Consider the projection map $e$ of $M$ onto $K$ with kernel $(1-e) M=L$. Then $e^{2}=e \in \operatorname{End}(M)$ and $e M=K$. By hypothesis, $e X \subseteq X$ and $(1-e) X \subseteq X$. Hence $e X=X \cap K$ and $(1-e) X=X \cap L$. Therefore $X=(X \cap K) \oplus(X \cap L)$. Now $(K+X) / X=(K \oplus(X \cap L)) / X$ and $(L+X) / X=(L \oplus(X \cap K)) / X$. Hence $M=K+X+L+X=$ $(K \oplus(X \cap L)]+L+X$ implies that $M / X=(K \oplus(X \cap L)) / X+(L+X) / X$. Since $[K \oplus(X \cap L)] \cap(L+X)=(X \cap L) \oplus(X \cap K)=X, M / X=$ $(K \oplus(X \cap L)) / X \oplus(L+X) / X$. Thus, by (1), $M / X$ is a $\tau_{\mathcal{T}}$-lifting module.

Now we investigate when a finite direct sum of $\tau_{\mathcal{T}}$-lifting modules is $\tau_{\mathcal{T}}$-lifting. We discuss the following example to show that there exists a torsion theory $\tau$ where for $\tau_{\mathcal{T}}$-lifting modules $M_{1}, M_{2}, M=M_{1} \oplus M_{2}$ is not $\tau_{\mathcal{T}}$-lifting.

Example 3.3. Let $\mathbb{Z}$ denote the ring of integers and consider the $\mathbb{Z}$-modules $M_{1}=$ $\mathbb{Z} / 2 \mathbb{Z}$ and $M_{2}=\mathbb{Z} / 8 \mathbb{Z}$ and $M=M_{1} \oplus M_{2}$. Let $\tau:=(\mathcal{T}, \mathcal{F})$ denote the torsion theory on Mod- $\mathbb{Z}$ where $\mathcal{T}=\{K \in \operatorname{Mod}-\mathbb{Z}$; for each $k \in K$ there exists a positive integer $t$ depending on $k$ with $\left.2^{t} k=0\right\}$. Since $M_{1}, M_{2}$ are hollow, they are lifting, in particular they are $\tau_{\mathcal{T}}$-lifting by Proposition 2.13. Let $N=(\overline{1}, \overline{2}) \mathbb{Z}$, then it does not contain any submodule as a direct summand of $M$. Hence $M$ is not an $\tau_{\mathcal{T}}$-lifting module.

Theorem 3.4. Let $\tau=(\mathcal{T}, \mathcal{F})$ be a hereditary torsion theory. If $M_{1}$ is a semisimple module and $M_{2}$ is a $\tau_{\mathcal{T}}$-lifting module such that $M_{1}$ is $M_{2}$-projective, then $M=$ $M_{1} \oplus M_{2}$ is $\tau_{\mathcal{T}}$-lifting.

Proof. Let $0 \neq N \leq M$. Let $K=M_{1} \cap\left(N+M_{2}\right)$. We divide the proof into two cases.

Case (i) $K \neq 0$. Then $M_{1}=K \oplus K_{1}$ for some submodule $K_{1}$ of $M_{1}$ and so $M=K \oplus K_{1} \oplus M_{2}=N+\left(M_{2} \oplus K_{1}\right)$. Hence $K$ is $M_{2} \oplus K_{1}$-projective. By [9, Lemma 4.47], there exists a submodule $N_{1}$ of $N$ such that $M=N_{1} \oplus\left(M_{2} \oplus K_{1}\right)$. We may assume $N \cap\left(M_{2} \oplus K_{1}\right) \neq 0$. Then $N \cap\left(L+K_{1}\right)=L \cap\left(N+K_{1}\right)$ for any submodule $L$ of $M_{2}$. Since $M_{2}$ is $\tau_{\mathcal{T}}$-lifting, there exists a submodule $X$ of $M_{2} \cap\left(N+K_{1}\right)=N \cap\left(M_{2} \oplus K_{1}\right)$ such that $M_{2}=X \oplus Y$ and $Y \cap\left(N+K_{1}\right)$ is $\tau$-small in $M_{2}$. Hence $M=\left(N_{1} \oplus X\right) \oplus\left(Y \oplus K_{1}\right)$. Since $N_{1} \oplus X \leq N$ and $N \cap\left(Y \oplus K_{1}\right)=Y \cap\left(N+K_{1}\right), N \cap\left(Y \oplus K_{1}\right)=Y \cap\left(N+K_{1}\right)$ is $\tau_{\mathcal{T}}$-small in $Y \oplus K_{1}$ by Lemma 2.1. So $M$ is $\tau_{\mathcal{T}}$-lifting.

Case (ii) $K=0$. Then $N \leq M_{2}$. Since $M_{2}$ is $\tau_{\mathcal{T}}$-lifting, there exists a submodule $X$ of $N$ such that $M_{2}=X \oplus Y$ and $N \cap Y$ is $\tau_{\mathcal{T}}$-small in $Y$ for some submodule $Y$ 
of $M_{2}$. Hence $M=X \oplus\left(M_{1} \oplus Y\right)$ and $N \cap\left(M_{1} \oplus Y\right)=N \cap Y$ is $\tau_{\mathcal{T}}$-small in $Y$. By Lemma 2.1, $N \cap\left(M_{1} \oplus Y\right) \ll_{\tau_{\mathcal{T}}} M_{1} \oplus Y$.

The following example shows that being hereditary torsion theory in Theorem 3.4 is essential.

Example 3.5. Let $R$ denote the ring of all upper triangular $2 \times 2$ matrices with entries in the field $F$. Let $I=\left(\begin{array}{cc}F & F \\ 0 & 0\end{array}\right)$ be an idempotent right ideal of $R$ and $\tau=\{N \in \operatorname{Mod}-R \mid N X=N\}$ be a torsion theory. If we take the submodule $A=\left(\begin{array}{cc}0 & F \\ 0 & 0\end{array}\right)$ of $I$, we can see that $A I=0$ and so $\tau$ is not hereditary torsion theory. Let $J=\left(\begin{array}{cc}0 & 0 \\ 0 & F\end{array}\right)$ and let $M=I \oplus J$. The only direct summand of $M$ contained in $J$ is $(0)$ submodule but $J /(0) \notin \mathcal{T}$.

Theorem 3.6. Let $\tau=(\mathcal{T}, \mathcal{F})$ be a hereditary torsion theory.

(1) If $M_{1}$ is a $\tau_{\mathcal{T}}$-lifting module and $M_{2}$ is a $\tau$-torsion module, $M=M_{1} \oplus M_{2}$ is $\tau_{\mathcal{T}}$-lifting.

(2) If $M_{1}$ is a semisimple module and $M_{2}$ is a $\tau$-torsion module, then $M=M_{1} \oplus M_{2}$ is $\tau_{\mathcal{T}}$-lifting

\section{Proof.}

(1) Let $N$ be a submodule of $M$. We consider the submodule $N \cap M_{1}$ of $M_{1}$. Since $M_{1}$ is a $\tau_{\mathcal{T}}$-lifting module, there exits a direct summand $K$ of $M_{1}$ ( in $M$ ) such that $\left(N \cap M_{1}\right) / K$ is $\tau_{\mathcal{T}}$-small in $M / K$ by Proposition 2.5 . Note that $N /\left(N \cap M_{1}\right) \cong\left(N+M_{1}\right) / M_{1} \in \mathcal{T}$ by assumption. This follows that $N / K$ is $\tau_{\mathcal{T}}$-small in $M / K$. By Lemma $2.1, M$ is $\tau_{\mathcal{T}}$-lifting.

(2) By (1).

The following example shows that being hereditary torsion theory in Theorem 3.6 is essential.

Example 3.7. Let $R$ denote the ring of all upper triangular $2 \times 2$ matrices with entries in the field $F$. Assume that $M=M_{1} \times M_{2}$ is an $R$-module, where $M_{1}=$ $\left(\begin{array}{cc}0 & F \\ 0 & F\end{array}\right)$ and $M_{2}=\left(\begin{array}{cc}F & F \\ 0 & 0\end{array}\right)$. Note that $M_{1}$ is semisimple and $M_{2}$ is a $\tau$ torsion module. Let $I=\left(\begin{array}{cc}F & F \\ 0 & 0\end{array}\right)$ be an idempotent right ideal of $R$ and $\tau=\{N \in$ $\operatorname{Mod}-R \mid N X=N\}$ be a torsion theory. Since $I$ is idempotent then it is $\tau$-torsion. We 
consider the submodule $I^{\prime}=\left(\begin{array}{cc}0 & F \\ 0 & 0\end{array}\right)$ of $I$. Since $I^{\prime} I=0, I^{\prime}$ is not $\tau$-torsion. But $M_{2} I=M_{2}$, this follows that $\tau$ is not hereditary. Let $N=\left(\begin{array}{cc}0 & F \\ 0 & 0\end{array}\right) \times\left(\begin{array}{cc}0 & F \\ 0 & 0\end{array}\right)$. Since $N I=0$, the module $N$ does not contain any direct summand $K$ of $M$ such that $N / K$ is $\tau$-small in $M / K$.

For a module $M$, let $\Gamma_{\mathcal{T}}(M)=\sum\left\{L \leq M \mid L\right.$ is a $\tau_{\mathcal{T}}$-small submodule of $\left.M\right\}$.

Lemma 3.8. Let $M$ be a module. Then

$$
\Gamma_{\mathcal{T}}(M)=\cap\{L \leq M \mid M / L \in \mathcal{T}, L \text { is maximal in } M\}
$$

Proof. Let $A=\cap\{L \leq M \mid M / L \in \mathcal{T}, L$ is maximal in $M\}$. It is easy to see that $\Gamma_{\mathcal{T}}(M) \leq A$. Conversely, assume that $a \in A$ such that $a R \nless_{\tau_{\mathcal{T}}} M$. Then the set

$$
\mathcal{F}=\{B \leq M \mid B \neq M, M / B \in \mathcal{T}, B+a R=M\}
$$

is non-zero. By Zorn's Lemma, $\mathcal{F}$ has a maximal element, say $B_{0}$. Now we claim that $B_{0}$ is a maximal submodule of $M$. If there exists $C \leq M$ such that $B_{0}<C<M$, then $M / C \in \mathcal{T}$ (since $M / B_{0} \in \mathcal{T}$ ) and $C+a R=M$. That means $C \in \mathcal{F}$, a contradicts the maximal of $B_{0}$. Thus $B_{0}$ is a maximal submodule of $M$ and $M / B_{0} \in \mathcal{T}$. This is a contradiction with $a \notin B_{0}$. Thus $a \in \Gamma_{\mathcal{T}}(M)$.

Theorem 3.9. The following are equivalent for a module $M$.

(1) $\Gamma_{\mathcal{T}}(M)$ is Artinian.

(2) Every $\tau_{\mathcal{T}^{-}}$-small submodule of $M$ is Artinian.

(3) M satisfies DCC on $\tau_{\mathcal{T}}$-small submodules.

Dually, the following are equivalent.

$\left(1^{\prime}\right) M$ has ACC on $\tau_{\mathcal{T}}$-small submodules of $M$.

$\left(2^{\prime}\right) \Gamma_{\mathcal{T}}(M)$ is Noetherian.

Proof. $\quad(1) \Rightarrow(2) \Rightarrow(3)$. They are clear.

$(3) \Rightarrow(1)$. It suffices to prove that any factor module of $\Gamma_{\mathcal{T}}(M)$ is finitely cogenerated. If there exists a factor module of $\Gamma_{\mathcal{T}}(M)$ that is not finitely cogenerated, then the set $\Omega=\left\{L \leq \Gamma_{\mathcal{T}}(M) \mid \Gamma_{\mathcal{T}}(M) / L\right.$ is not finitely cogenerated $\}$ is nonempty. Let $\left\{L_{\lambda}: \lambda \in\right.$ $\Lambda\}$ be any chain of submodules in $\Omega$. Let $L=\bigcap_{\lambda \in \Lambda} L_{\lambda}$. If $L \notin \Omega$, then $\Gamma_{\mathcal{T}}(M) / L$ is finitely cogenerated and hence $L=L_{\lambda}$ for some $\lambda \in \lambda$. It follows that $L \in \Omega$, a contradiction. Thus $L \in \Omega$. By Zorn's Lemma, $\Omega$ has a minimal member, say $A$. Let $N$ be a finitely generated submodule of $\Gamma_{\mathcal{T}}(M)$. Then $N$ is a $\tau_{\mathcal{T}}$-small submodule of $M$ and hence Artinian by hypothesis. That means $\Gamma_{\mathcal{T}}(M)$ is a locally Artinian module. 
Now let $x \in \Gamma_{\mathcal{T}}(M), x \notin A$. Then $x R$ is Artinian and $(x R+A) / A \simeq x R /(x R \cap A)$. So $(x R+A) / A$ is a nonzero Artinian module and hence $\Gamma_{\mathcal{T}}(M) / A$ has essential socle. Let $S$ denote the submodule of $\Gamma_{\mathcal{T}}(M)$, containing $A$, such that $S / A$ is the socle of $\Gamma_{\mathcal{T}}(M) / A$. Thus $S / A$ is not finitely generated by [1, Proposition 10.7].

Next we show that $A \ll_{\tau_{\mathcal{T}}} M$. If $M=A+B$ with $M / B \in \mathcal{T}$, then $S=A+(S \cap$ $B)$. Suppose that $A \cap B \neq A$. Then $\Gamma_{\mathcal{T}}(M) /(A \cap B)$ is finitely cogenerated by the choice of $A$. But $S / A=(A+(S \cap B)) / A \simeq(S \cap B) /(A \cap B) \leq S o c\left(\Gamma_{\mathcal{T}}(M) /(A \cap B)\right)$ and hence $S / A$ is finitely generated. This is a contradiction. Thus $A=A \cap B \leq B$ and we have $M=A+B=B$. So $A \ll_{\tau_{\mathcal{T}}} M$.

Now suppose that $M=S+V$ for some submodule $V$ of $M$ and $M / V \in \mathcal{T}$. Then $M /(A+V)=(S+V) /(A+V) \simeq S /(A+(S \cap V))$. Thus $M /(A+V)$ is semisimple. If $M \neq A+V$, then there exists a maximal submodule $W$ of $M$ such that $A+V \leq W$. Note that $M / W \simeq(M / V) /(W / V), M / V \in \mathcal{T}$ and hence $M / W \in \mathcal{T}$. It follows that $\Gamma_{\mathcal{T}}(M) \leq W$ by Lemma 3.8. Then this gives the contradiction $M=W$. Thus $M=A+V$, hence $M=V$ since $A \ll_{\tau_{\mathcal{T}}} M$. Therefore $S \ll_{\tau_{\mathcal{T}}} M$ and hence $S$ is Artinian by hypothesis. It follows that $S / A$ is Artinian, and, in particular, $S / A$ is finitely generated. This is a contradiction. Thus $\Gamma_{\mathcal{T}}(M)$ is Artinian.

$\left(1^{\prime}\right) \Rightarrow\left(2^{\prime}\right)$ We assume that $X_{1}<X_{2}<\cdots$ be a strictly ascending chain of submodules of $\Gamma_{\mathcal{T}}(M)$. Let $x_{1} \in X_{1}$ and $x_{i} \in X_{i}-X_{i-1}$ for $i \geq 2$. Clearly, $x_{1} R \leq x_{1} R+x_{2} R \leq$ $\cdots$ and each $x_{i} R$ is $\tau_{\mathcal{T}}$-small. Hence, by Lemma 2.1, for each $n, \sum_{i=1}^{n} x_{i} R$ is $\tau_{\mathcal{T}}$-small submodule of $M$. This follows that $M$ does not have ACC on $\tau_{\mathcal{T}}$-small submodules, contradiction.

$\left(2^{\prime}\right) \Rightarrow\left(1^{\prime}\right)$ Clear.

In case $\tau_{*}=\left(\mathcal{T}_{*}, \mathcal{F}_{*}\right)$ is a Goldie torsion theory, the notion $\Gamma_{\mathcal{T}_{*}}(M)$ is defined similarly.

Theorem 3.10. Let $\tau_{*}=\left(\mathcal{T}_{*}, \mathcal{F}_{*}\right)$ be a Goldie torsion theory and let $M$ be a countably $\tau_{\mathcal{T}_{*}}$-lifting almost $\tau$-torsion module. If $\Gamma_{\mathcal{T}_{*}}(M)$ is $\tau_{\mathcal{T}_{*}}$-small in $M$, then $M$ is isomorphic to a direct sum of cyclic submodules.

Proof. Let $\left\{x_{1}, x_{2}, \cdots\right\}$ be a generating set for $M$. Since $M$ is $\tau_{\mathcal{T}_{*}}$-lifting module, we have a decomposition $M=A_{1} \oplus B_{1}$ such that $A_{1} \leq R x_{1}$ and $B_{1} \cap R x_{1} \ll_{\tau_{\mathcal{T}_{*}}} M$. Clearly, $A_{1}$ is cyclic. Let $C_{1}=B_{1} \cap R x_{1}$. By induction on a positive integer $n$, we can write $M=\left(\sum_{i=1}^{n} A_{i}\right) \oplus B_{n}$ such that $\sum_{i=1}^{n} R x_{i} \leq \oplus_{i=1}^{n} A_{i}+C_{i}$ and $C_{i} \ll_{\tau_{\mathcal{T}_{*}}} M$. By Proposition 2.11(2), the direct summand $B_{n}$ of $M$ is $\tau_{\tau_{*}}$-lifting. Then we have a decomposition $B_{n}=A_{n+1} \oplus B_{n+1}$ such that $A_{n+1} \leq R x_{n+1}$ and $B_{n+1} \cap R x_{n+1} \ll_{\tau_{\mathcal{T}_{*}}}$ $B_{n}$. Let $D=B_{n+1} \cap R x_{n+1}$. Then $M=\left(\sum_{i=1}^{n+1} A_{i}\right) \oplus B_{n+1}$ such that $\sum_{i=1}^{n+1} R x_{i} \leq$ $\oplus_{i=1}^{n+1} A_{i}+C_{n}+D$ and $C_{n}+D \ll_{\tau_{\mathcal{T}_{*}}} M$. Since $C=\sum_{i \in \mathbb{N}} C_{i} \leq \Gamma_{\mathcal{T}_{*}}(M)$ and $\Gamma_{\mathcal{T}_{*}}(M)$ is $\tau_{\mathcal{T}_{*}}$-small in $M$ then there exits a projective semisimple submodule $P$ of $C$ such that $M=\sum_{i \in \mathbb{N}} R x_{i}=\left(\oplus_{i \in \mathbb{N}} A_{i}\right) \oplus C=\left(\oplus_{i \in \mathbb{N}} A_{i}\right) \oplus P$ by Lemma 2.1.

Let $M$ be a $\delta$-supplemented module. In [5, Lemma 2.12], Koşan proved that 
$M / \delta(M)$ is semisimple.

Lemma 3.11. Let $M$ be a $\tau_{\mathcal{T}}$-supplemented module. Then $M / \Gamma_{\mathcal{T}}(M)$ is a semisimple module.

Proof. Let $\Gamma_{\mathcal{T}}(M) \leq N \leq M$. There exists $X \leq M$ such that $M=N+X$ and $N \cap X \ll_{\tau_{\mathcal{T}}} X$. So $N \cap X \ll_{\tau_{\mathcal{T}}} M$. Then $M / \Gamma_{\mathcal{T}}(M)=N / \Gamma_{\mathcal{T}}(M)+(X+$ $\left.\Gamma_{\mathcal{T}}(M)\right) / \Gamma_{\mathcal{T}}(M)=N / \Gamma_{\mathcal{T}}(M) \oplus\left(X+\Gamma_{\mathcal{T}}(M)\right) / \Gamma_{\mathcal{T}}(M)$ because $N \cap\left(X+\Gamma_{\mathcal{T}}(M)\right)=$ $(N \cap X)+\Gamma_{\mathcal{T}}(M)=\Gamma_{\mathcal{T}}(M)$.

A module $M$ is called an amply $\tau_{\mathcal{T}}$-supplemented if for any submodules $A, B$ of $M$ with $M=A+B$, there exists a $\tau_{\mathcal{T}}$-supplement $P$ of $A$ such that $P \leq B$.

Theorem 3.12. Let $M$ be a module. Then $M$ is Artinian if and only if $M$ is an amply $\tau_{\mathcal{T}}$-supplemented module and satisfies DCC on $\tau_{\mathcal{T}}$-supplement submodules and on $\tau_{\mathcal{T}}$-small submodules.

Proof. The necessary condition is clear. Conversely, assume that $M$ is an amply $\tau_{\mathcal{T}}$-supplemented module which satisfies DCC on $\tau_{\mathcal{T}}$-supplement submodules and on $\tau_{\mathcal{T}}$-small submodules. Then $\Gamma_{\mathcal{T}}(M)$ is Artinian by Theorem 3.9. Next, it suffices to show that $M / \Gamma_{\mathcal{T}}(M)$ is Artinian. It is clear that $M / \Gamma_{\mathcal{T}}(M)$ is semisimple by Lemma 3.11 .

Now suppose that $\Gamma_{\mathcal{T}}(M) \leq N_{1} \leq N_{2} \leq N_{3} \leq \cdots$ is an ascending chain of submodules of $M$. Because $M$ is an amply $\tau_{\mathcal{T}}$-supplemented module, there exists a descending chain of submodules $K_{1} \leq K_{2} \leq \cdots$ such that $K_{i}$ is a $\tau_{\mathcal{T}}$-supplement of $N_{i}$ in $M$ for each $i \geq 1$. By hypothesis, there exists a positive integer $t$ such that $K_{t}=$ $K_{t+1}=K_{t+2}=\cdots$. Because $M / \Gamma_{\mathcal{T}}(M)=N_{i} / \Gamma_{\mathcal{T}}(M) \oplus\left(K_{i}+\Gamma_{\mathcal{T}}(M)\right) / \Gamma_{\mathcal{T}}(M)$ for all $i \geq t$, it follows that $N_{t}=N_{t+1}=\cdots$. Thus $M / \Gamma_{\mathcal{T}}(M)$ is Noetherian, and hence finitely generated. So $M / \Gamma_{\mathcal{T}}(M)$ is Artinian, as desired.

Corollary 3.13. Let $M$ be a finitely generated. Then $M$ is Artinian if and only if $M$ is a $\tau_{\mathcal{T}}$-supplemented module satisfies DCC on $\tau_{\mathcal{T}}$-small submodules.

Proof. Since $M / \Gamma_{\mathcal{T}}(M)$ is semisimple and $M$ is finitely generated, $M / \Gamma_{\mathcal{T}}(M)$ is Artinian. Now that $M$ satisfies DCC on $\tau_{\mathcal{T}}$-small submodules, $\Gamma_{\mathcal{T}}(M)$ is Artinian. Thus $M$ is Artinian.

Corollary 3.14. If $M$ is a $\tau_{\mathcal{T}}$-supplemented module and satisfies DCC on $\tau_{\mathcal{T}^{-}}$-small submodules, then so does $M / A$ for any submodule $A$ of $M$.

Proof. $\quad$ Let $A$ be any submodule of $M$ and $B_{1} / A \leq B_{2} / A \leq \cdots$ where each $B_{i} / A \ll_{\tau_{\mathcal{T}}} M / A$. Let $C$ be an $\tau_{\mathcal{T}}$-supplement of $A$ in $M$. Then $M / A=(A+C) / A \simeq$ $C / A \cap C$. Since $B_{i} / A$ is $\tau_{\mathcal{T}}$-small in $M / A, B_{i} / A \simeq D_{i} / A \cap C \ll_{\tau_{\mathcal{T}}} C / A \cap C$ for some $D_{i}$. Next we prove that $D_{i} \ll_{\tau_{\mathcal{T}}} M$. Let $D_{i}+E=M$ with $M / E \in \mathcal{T}$. Then $\left(D_{i}+(E+A \cap C)\right) / A \cap C=M / A \cap C$. Hence $E+A \cap C=M$ and $E=M$. 
Thus we have $D_{1} \leq D_{2} \leq \cdots$. Since $M$ satisfies ACC on $\tau_{\mathcal{T}}$-small submodules, there exists $\mathrm{n}$ such that $D_{k}=D_{k+1}$ for all $k \geq n$. Thus $B_{k} / A=B_{k+1} / A$ for all $k \geq n$. Therefore $M / A$ satisfies ACC on $\tau_{\mathcal{T}}$-small submodules, as required.

A submodule $N$ of $M$ is called $\mathcal{T}$-semimaximal if $N=\cap_{i=1}^{n} L_{i}$ with $L_{i}$ is maximal in $M$ and $M / L_{i} \in \mathcal{T}$ for any $i=1, \ldots, n$.

Theorem 3.15. Let $M$ be a module. Then the following statements are equivalent:

(1) $M$ is Artinian.

(2) $M$ satisfies DCC on $\tau_{\mathcal{T}}$-small submodules and on $\mathcal{T}$-semimaximal submodules.

(3) $M$ satisfies DCC on $\tau_{\mathcal{T}}$-small submodules and $\Gamma_{\mathcal{T}}(M)$ is a $\mathcal{T}$-semimaximal submodule of $M$.

Proof. $\quad(1) \Rightarrow(2)$. It is clear.

(2) $\Rightarrow(3)$. Assume that $M$ satisfies DCC on $\mathcal{T}$-semimaximal submodules. Let $N$ be a minimal $\mathcal{T}$-semimaximal submodule of $M$. Then $\Gamma_{\mathcal{T}}(M) \leq N$. If $M=\Gamma_{\mathcal{T}}(M)$, then $\Gamma_{\mathcal{T}}(M)=N$, a contradiction. Suppose that $M \neq \Gamma_{\mathcal{T}}(M)$. If $P$ is a maximal submodule of $M$ and $M / P \in \mathcal{T}$, then $N \cap P$ is an $\mathcal{T}$-semimaximal submodule of $M$ and hence $N=N \cap P$ or $N \leq P$. It follows that $N \leq \Gamma_{\mathcal{T}}(M)$. Therefore $N=\Gamma_{\mathcal{T}}(M)$. Thus $\Gamma_{\mathcal{T}}(M)$ is an $\mathcal{T}$-semimaximal submodule of $M$.

(3) $\Rightarrow(1)$. It is clear $\Gamma_{\mathcal{T}}(M)$ is Artinian. If $M=\Gamma_{\mathcal{T}}(M)$, then $M$ is Artinian. Suppose that $M \neq \Gamma_{\mathcal{T}}(M)$. Then $\Gamma_{\mathcal{T}}(M)=P_{1} \cap P_{2} \cap \cdots \cap P_{n}$, where $P_{i}$ is a maximal submodule of $M$ and $M / P_{i} \in \mathcal{T}$ for any $i=1, \ldots, n$. It follows that $M / \Gamma_{\mathcal{T}}(M)$ embeds in the finitely generated semisimple module $M / P_{1} \oplus \cdots \oplus M / P_{n}$. Hence $M / \Gamma_{\mathcal{T}}(M)$ is Artinian. It follows that $M$ is Artinian.

\section{4. $\tau_{c G}$-LiFTing Modules}

In this section we investigate lifting modules relative to dual Goldie torsion theory $\tau_{c G}$ on Mod- $R$. In [13], Talebi and Vanaja defined, $\bar{Z}(M)$ as follows:

$$
\bar{Z}(M)=\operatorname{Re}(M, \mathcal{S})=\bigcap\{\operatorname{Ker}(g) \mid g \in \operatorname{Hom}(M, L), L \in \mathcal{S}\},
$$

where $\mathcal{S}$ denotes the class of all small modules. They called $M$ a cosingular (noncosingular) module if $\bar{Z}(M)=0(\bar{Z}(M)=M)$.

In [11], Ramamurthi defined the dual Goldie Torsion theory as that generated by the class of small modules and studied some of its properties. Further study was done by $\ddot{O}$ zcan and Harmanci [10]. In [13], Talebi and Vanaja defined torsion theory cogenerated by the class $\mathcal{S}$ of all small modules for a fixed $M \in$ Mod- $R$. We denote this theory by $\tau_{c G}:=\left(\mathcal{T}_{\mathcal{S}}, \mathcal{F}_{\mathcal{S}}\right)$.

Proposition 4.1. Let $M$ be an R-module. Then 
(1) The torsion class $\mathcal{T}_{\mathcal{S}}$ is the class of all noncosingular modules;

(2) The torsion free class $\mathcal{F}_{\mathcal{S}}$ is the class of modules for which every nonzero submodule is not noncosingular;

(3) $\tau_{c G}(M)$ is its largest noncosingular submodule.

Proof. See [13, Proposition 3.1].

Theorem 4.2. If $M$ is amply supplemented and $\tau_{c G \mathcal{F}_{\mathcal{S}}}$-lifting, then $\tau_{c G}(M)$ is a direct summand of $M$.

Proof. If $M$ is a $\tau_{c G \mathcal{F}_{\mathcal{S}}}$-lifting module with $\tau_{c G}(M)=0$, then by Example 2.10, $M$ is lifting. Clearly, $\tau_{c G}(M)$ is noncosingular, therefore $\tau_{c G}(M)$ is coclosed in $M$ and so is direct summand. So suppose that $M$ is not $\tau_{c G}$-torsion free. If $K$ is a $\tau_{c G \mathcal{F}_{\mathcal{S}}}{ }^{-}$ supplement of $\tau_{c G}(M)$, then $M=\tau_{c G}(M)+K$ and $\tau_{c G}(M) \cap K \ll_{\tau_{c G} \mathcal{F}_{\mathcal{S}}} K$. Thus $\tau_{c G}\left(\tau_{c G}(M) \cap K\right)=0$, therefore $\tau_{c G}(M) \cap K$ is cosingular and so it is a $\tau_{c G}(M)$ torsion free submodule. On the other hand, since $\tau_{c G}\left(M / \tau_{c G}(M)\right)=0$, we have $M / \tau_{c G}(M) \in \mathcal{F}$. But

$$
M / \tau_{c G}(M)=\left(K+\tau_{c G}(M)\right) / \tau_{c G}(M) \simeq K /\left(\tau_{c G}(M) \cap K\right) .
$$

Thus $K /\left(\tau_{c G}(M) \cap K\right) \in \mathcal{F}$. Since $K \in \mathcal{F}$, we can obtain that $K$ is a direct summand of $M$. Let $M=K \oplus K^{\prime}$ for some $K^{\prime}$ of $M$. Now we show that $M / K$ is a $\tau_{c G}(M)$ torsion module. Note that

$$
M / K=\left(K+\tau_{c G}(M)\right) / K \simeq \tau_{c G}(M) /\left(\tau_{c G}(M) \cap K\right)
$$

as $\tau_{c G}(M) \in \mathcal{T}$. Hence $\tau_{c G}(M) /\left(\tau_{c G}(M) \cap K\right) \in \mathcal{T}$, and so $M / K$ is $\tau_{c G}(M)$-torsion. Moreover $M / K \simeq K^{\prime}$, and so $K^{\prime}$ is a $\tau_{c G}(M)$-torsion submodule. But

$$
\tau_{c G}(M)=\tau_{c G}(K) \oplus \tau_{c G}\left(K^{\prime}\right)=\tau_{c G}\left(K^{\prime}\right)=K^{\prime} .
$$

Therefore $M=K \oplus \tau_{c G}(M)$.

Let $\mathcal{X}$ and $\mathcal{Y}$ be classes of modules. We write $\mathcal{X} \leq \mathcal{Y}$ in case every object of $\mathcal{X}$ is in $\mathcal{Y}$.

Lemma 4.3. ([7, Lemma 2.5] ). Let $\mathcal{X}$ and $\mathcal{Y}$ be classes of modules with $\mathcal{X} \leq \mathcal{Y}$. Then every $\mathcal{Y}$-lifting module is $\mathcal{X}$-lifting.

The torsion theories on Mod- $R$ may be partially ordered. If $\tau_{1}=\left(\mathcal{T}_{1}, \mathcal{F}_{1}\right)$ and $\tau_{2}=\left(\mathcal{T}_{2}, \mathcal{F}_{2}\right)$ are two torsion theories, we say that $\tau_{1}$ is smaller than $\tau_{2}\left(\tau_{1} \leq \tau_{2}\right)$ if and only if $\mathcal{F}_{1} \supseteq \mathcal{F}_{2}$ (equivalently $\mathcal{T}_{1} \subseteq \mathcal{T}_{2}$ ).

Proposition 4.4. Let $\tau=\left(\mathcal{T}_{1}, \mathcal{F}_{1}\right)$ and $\varrho=\left(\mathcal{T}_{2}, \mathcal{F}_{2}\right)$ be torsion theories such that $\tau \leq \varrho$. If module $M$ is $\varrho \mathcal{T}_{2}$-lifting, then $M$ is $\tau_{\mathcal{T}_{1}}$-lifting. 
Proof. By Lemma 4.3.

Theorem 4.5. Suppose that $\tau=(\mathcal{T}, \mathcal{F})$ is a torsion theory such that $\tau_{c G} \leq \tau$. Then a noncosingular module $M$ is $\tau_{\mathcal{T}}$-lifting if and only if it is $\tau_{c G \mathcal{T}_{\mathcal{S}}}$-lifting.

Proof. Since $\tau_{c G} \leq \tau$, by Proposition 4.4, if $M$ is $\tau_{\mathcal{T}}$-lifting, then $M$ is $\tau_{c G \mathcal{T}_{\mathcal{S}}}$ lifting. Conversely, suppose that $M$ is $\tau_{c G \mathcal{T}_{\mathcal{S}}}$-lifting. Let $N$ be a submodule of $M$. Then there exists a decomposition $N=A \oplus B$ such that $A$ is a direct summand of $M$ and $B \ll_{\tau_{c G}} M$. Let $M=B+Y$ such that $M / Y$ is $\tau$-torsion. Since $M$ is noncosingular, $\bar{Z}(M)=M$. So $M$ is $\tau_{c G}$-torsion. Thus $M / Y$ is $\tau_{c G}$-torsion. Therefore $Y=M$. Hence $B \ll_{\tau} M$, and $M$ is $\tau_{\tau}$-lifting.

\section{Examples 4.6.}

(1) Let $\mathcal{X}=\{X \in M o d-\mathbb{Z}: X 2=0\}$ and $\mathcal{Y}=\{Y \in M o d-\mathbb{Z}: Y 4=0\}$. We consider the $\mathbb{Z}$-module $M=(\mathbb{Z} / 2 \mathbb{Z}) \oplus(\mathbb{Z} / 8 \mathbb{Z})$. It is easy to see that $\mathcal{X} \leq \mathcal{Y}$ and $M$ is $\mathcal{X}$-lifting but is not $\mathcal{Y}$-lifting.

(2) The $\mathbb{Z}$-module $\mathbb{Q}$, the set of all rational numbers, is noncosingular by [13, Remark 2.11]. So $\mathbb{Q}$ is $\tau_{c G}$-torsion. Now Example 2.10 shows that $\mathbb{Q}$ is $\tau_{c G}$-lifting. But $\mathbb{Q}_{\mathbb{Z}}$ is not lifting.

(3) Let $\tau$ be a torsion theory such that $\tau_{c G} \leq \tau$. Then

(i) Every noncosingular module is $\tau_{c G}$-lifting and $\tau$-lifting.

(ii) Every $\tau_{c G}$-lifting module is $\tau$-lifting.

\section{REFERENCES}

1. F. W. Anderson and K. R. Fuller, Rings and Categories of Modules, Springer-Verlag, New York, 1974.

2. S. Crivei, Relatively lifting modules, Algebra Colloquium, Spec. Iss., 17(1) (2010), 789798.

3. J. S. Golan, Torsion Theories, Pitmann Mono. and Surveys in Pure and Appl. Math., 29, (1986).

4. D. Keskin, On lifting modules, Comm. Algebra, 28(7) (2000), 3427-3440.

5. M. T. Koşan, $\delta$-lifting and $\delta$-supplemented Modules, Algebra Colloquium, 14(1) (2007), 53-60.

6. M. T. Koşan, $\tau$-Supplemented modules and $\tau$-weakly supplemented modules, Archivum Mathematicum, 43 (2007), 251-257.

7. M. T. Koşan and A. Harmanci, Modules which are lifting relative to module classes, Kyungpook J. Math., 48(1) (2008), 63-71.

8. M. T. Koşan, Module classes and the lifting property, Turkish J. Math., 35(3) (2011), 379-389. 
9. S. H. Mohamed and B. J. Müller, Continuous and Discrete Modules, London Math. Soc. LNS 147 Cambridge Univ. Press, Cambridge, 1990.

10. A. Ç. Özcan and A. Harmanci, Characterization of some rings by functor $Z^{*}($.$) , Turkish$ J. Math., 21 (1997), 325-331.

11. V. S. Ramamurthi, The smallest left exact radical containing the Jacobson radical, Ann. Soc. Sci. Bruxelles Ser., 96(I) (1982), 201-206.

12. B. Stenström, Rings of Quotients, Springer-Verlag, 1975.

13. Y. Talebi and N. Vanaja, The torsion theory cogenerated by M-small modules, Comm. Algebra, 30 (2002), 1449-1460.

14. R. Wisbauer, Foundations of Module and Ring Theory, Gordon and Breach, Reading, 1991.

15. Y. Zhou, Generalizations of perfect, semiperfect, and semiregular rings, Algebra Colloquium, 7(3) (2000), 305-318.

M. Tamer Koşan

Department of Mathematics

Gebze Institute of Technology

Gebze-Kocaeli, Turkey

E-mail: mtkosan@gyte.edu.tr

Truong Cong Quynh

Department of Mathematics

Danang University

459 Ton Duc Thang, Vietnam

E-mail: tcquynh@dce.udn.vn

tcquynh@live.com

Yahya Talebi

Department of Mathematics

Faculty of Basic Science

University of Mazandaran

Babolsar, Iran

E-mail: talebi@umz.ac.ir 